\title{
LA VARIACIÓN DE /@ ${ }^{\mathrm{s}}$ /: ESTUDIO COMPARATIVO DE DOS MUESTRAS DE POBLACIÓN GRANADINAS
}

\author{
Elisabeth MELguizo MorenO \\ Universidad de Granada \\ ely@ugr.es
}

\begin{abstract}
Resumen
En este trabajo analizamos los resultados que nos ofrece el estudio comparativo de dos muestras de población: la constituida por hablantes nacidos y residentes en la localidad de Pinos Puente y la formada por sujetos procedentes de dicho municipio que se han instalado definitivamente en Granada (inmigrantes pineros). Nuestro propósito es estudiar el grado de acomodación de habla que manifiestan los hablantes en la capital granadina mediante la distribución que experimentan dos normas de funcionamiento lingüístico: la de reducción (seseoceceo) y la de distinción en las muestras recogidas. Los parámetros seleccionados para su estudio son la edad, la educación y el sexo. Precisamente en este terreno se adentra el Proyecto de Investigación sobre la Formación de Dialectos (FORDIAL) que proporciona datos relevantes sobre la formación de variedades lingüísticas que surgen de los contactos que se producen en los núcleos urbanos, como consecuencia de los movimientos poblacionales ligados a áreas rurales. En nuestro caso particular interesa principalmente cómo se produce el abandono del ceceo (norma vernácula) a favor de la distinción (variedad de prestigio en Granada).

PALABRAS CLAVE: sociolingüística, variación, acomodación.
\end{abstract}

\begin{abstract}
In this paper we analyze the results that the comparative study offers from two samples of population: the one formed by speakers born and raised in the town of Pinos Puente and the one formed by subjects coming from such town who have definitely settled in Granada (pineros immigrants). Our purpose is to study the degree of speech accommodation that is manifested by speakers living in Granada city through the distribution that two rules of linguistic functioning manifest: the one of reduction and the one of distinction in the collected samples. The selected parameters for its study are age, education and sex. Precisely on that matter it's what the Research project about Dialect formation is about, which provides relevant data about the formation of linguistic varieties that are born from the contacts which take place in urban areas, as a consequence of migration movements related to rural areas. In our particular case we are interested mainly on how the abandon of ceceo takes place (vernacular norm) in favour of the distinction (prestige variety in Granada).
\end{abstract}

KEYWORDS: sociolinguistics, variation, accommodation.

\section{1. objetivos}

Se pretende poner de manifiesto el desarrollo de los procesos de acomodación de habla que se producen como consecuencia del trasiego de población de un área rural (Pinos Puente) a un núcleo urbano (Granada). Concretamente analizamos las pautas de variación de las estridentes posterior $/ \mathrm{s} / \mathrm{y}$ anterior $/ \theta /$. La identificación de los individuos con dichas pautas de pronunciación, sin embargo, tiende a quedar neutralizada en función del nivel educacional, sobre todo, la edad y el sexo. La valoración que los hablantes conceden a las 
formas lingüísticas hacen que se consideren poco prestigiosas las normas de pronunciación estridente (seseo) o mate (ceceo) y muy prestigioso el fenómeno de distinción $/ \mathrm{s} /: / \theta /{ }^{1}$.

No obstante, el prestigio social de que goza una variable lingüística depende de condicionamientos no sólo sociales sino también individuales. Así, encontramos hablantes residentes en el pueblo con actitudes sociolingüísticas opuestas: la inmensa mayoría muestra su variedad vernácula (ceceo), con independencia de la edad o el género, ya que su lugar de origen se halla en un área de ceceo (Pinos Puente); el resto, sin embargo, se distinguen por tratar de adquirir la distinción fonológica /s/:/ $/$ /. Estos sujetos se percatan de la valoración negativa que tiene su ceceo originario y tratan de abandonarlo, en beneficio de la norma que goza de mayor aceptación social (distinción s/ $\theta$ ). En ese intento, los inmigrantes pineros cometen errores puesto que carecen de la dorsal/s/ en su sistema fonológico ${ }^{2}$.

Cuando estos individuos emigran a la capital granadina siguen manifestando actitudes opuestas: unos, favorecen la presencia de un prestigio encubierto ('covert prestige'), es decir, siguen siendo ceceantes, en función de la solidaridad con su pueblo de origen, su lealtad local, de las relaciones informales con los amigos, parientes, vecinos, etc. Constituyen, pues, una red social densa y una comunidad cerrada al exterior, lo cual permite la conservación de estructuras del vernacular de origen. Suelen ser individuos de la tercera generación sin estudios o jóvenes sin ningún grado de instrucción formal; otros, adoptan una actitud proclive al cambio y se benefician del prestigio abierto ('overt prestige') de que goza la norma culta granadina (distinción /s/:/ / /). Son jóvenes universitarios o hablantes de la tercera generación con un alto grado de instrucción formal. Todos ellos optan por la distinción aunque a veces subyacen sonidos intermedios de $/ \mathrm{s} / / / \theta /$ e incluso alófonos seseantes en su intento de acomodación a la distinción como norma más valorada en Granada.

El citado proceso se analiza a través de un estudio cuantitativo de la variable $/ \theta^{\mathrm{s}} /$ en el habla de los individuos de dos calas de población íntimamente relacionadas: una, ubicada en Pinos Puente y otra, constituida por hablantes procedentes de Pinos Puente que han fijado su residencia en Granada (inmigrantes pineros).

La edad, el sexo y el nivel educativo determinarán las pautas de funcionamiento de dos normas de pronunciación: (a) una vernácula, ligada al contraste fónico estridente (seseo) / mate (ceceo) y, (b) una norma general prestigiosa (la distinción).

\footnotetext{
${ }^{1}$ En las dos calas poblacionales analizadas interesan especialmente dos fenómenos: el ceceo y la distinción. El primero porque constituye la norma vernácula de los hablantes de nuestro estudio; y la distinción de /s/: / $\theta /$ ya que es la variedad lingüística, junto con el seseo, que, en la actualidad, goza de prestigio social en Granada (Moya y García Wiedemann, 1995: 55\% distinción, 40\% seseo y 5\% ceceo) y, en consecuencia, la que tratan de adquirir los inmigrantes pineros que se instalan en la capital.

${ }^{2}$ Con esta afirmación queremos decir que a los hablantes les supone un esfuerzo enorme articular la sibilante pero no que desconozcan su existencia. Evidentemente, todos distinguen los fonemas /s/: / $\theta$ / en la escritura.

${ }^{3}$ Más adelante mostraremos el influjo del prestigio social sobre el continuum de variación que representan los hablantes de la comunidad de habla estudiada.
} 


\section{Datos}

Los datos de este trabajo proceden de un corpus oral basado en la realización de entrevistas con una duración de 30 o 45 minutos según el número de personas encuestadas (una o dos, respectivamente) y de un corpus escrito obtenido a través de cuestionarios sociológicos y reticulares que proporcionaban información personal del individuo y de su red inmediata 4 .

La población estudiada está formada por dos calas: una ubicada en el pueblo (Pinos Puente) y otra en Granada (inmigrantes de procedencia rural). El total de informantes son $\mathrm{N}$ $=144(\mathrm{H}=72$ y $\mathrm{M}=72)$ para las dos muestras diseñadas, cada una de las cuales tiene un total de $\mathrm{N}=72$ hablantes $(\mathrm{H}=36 ; \mathrm{M}=36)$ : rurales (Pinos Puente) e inmigrantes instalados. Para su estudio se consideran tres generaciones de edad: $1^{\mathrm{a}}$ Generación: 15-24 años; $2^{\mathrm{a}}$ Generación: 25-54 años y $3^{\mathrm{a}}$ Generación: > 54 años; y tres niveles educativos: a) Sin estudios (0-6 años); b) Estudios Medios (7-11 años) y c) Estudios Superiores (Más de 11 años). La edad y el nivel educacional constituyen dos variables fundamentales para la estratificación de la muestra.

Hemos seleccionado ambas muestras por varios motivos: (a) la investigadora pertenece a la comunidad de habla estudiada, por tanto, el acceso al estilo de habla más natural y espontáneo (Labov, 1984; Milroy, 1987) resultaba una tarea fácil; (b) aprovechamos esta circunstancia para averiguar cómo se produce la acomodación lingüística de unos hablantes de procedencia rural en un área urbana (Granada); (c) asimismo, intentamos acceder a la variación inter- e intrapersonal de nuestros hablantes. Observamos que la adquisición de la distinción granadina está determinada por varios factores: educación, edad, contacto con el pueblo, tiempo de residencia en la ciudad, etc. No presenta tantos problemas la norma reductora ceceante que manifiestan los hablantes del pueblo ya que constituye su variedad de origen. Por consiguiente, decidimos adentramos en este estudio mediante un muestreo por cuotas con afijación uniforme (López Morales, 1994), en el cual, las variables de estratificación son la edad, el nivel educacional y el sexo. Se establece una división del espacio en estratos, en función de las variables de estratificación y se asigna igual número de hablantes a cada cuota (para cada sexo se seleccionan cuatro hablantes en cada casilla).

\section{Variables}

Hemos de distinguir entre variables sociodemográficas y variables lingüísticas.

\subsection{Variables sociodemográficas}

Las variables que se han considerado en este estudio son las siguientes:

\footnotetext{
${ }^{4}$ En dicho cuestionario había preguntas relativas a la edad, sexo, nivel educativo, años de estudio, ingresos, exposición a los media, barrio de residencia, ocupación, matrimonio, nacimiento de la pareja, satisfacción con la red, personas con las que trata, densidad, multiplicidad, ruralidad, modernidad, etc.
} 
- Edad. Los grupos generacionales mencionados ( $1^{\mathrm{a}}$ generación: 15-24 años; $2^{\mathrm{a}}$ generación: 25-54 años y $3^{\mathrm{a}}$ generación: $>54$ años) determinan el avance o retroceso de los fenómenos analizados.

- Educación. El grado de instrucción de los individuos resulta fundamental para establecer una comparación entre la probabilidad de ceceo (PC) y distinción (PD) en los hablantes del pueblo y de Granada. Los seis niveles educativos iniciales que aparecían en los cuestionarios, se recodificaron en tres grupos: sin estudios, estudios medios y estudios superiores.

- Sexo. Se establece una división genérica entre hombres y mujeres, con el fin de comprobar las diferencias entre ambos grupos.

\subsection{Variables lingüísticas}

El estudio de la variación de $/ \theta^{\mathrm{s}} /$ en las comunidades de habla estudiadas resulta complejo puesto que en ellas coexisten patrones de comportamiento variados. Las normas ideales de seseo, ceceo y distinción se distribuyen de manera irregular entre los informantes de cada cala poblacional.

Los hablantes granadinos (rurales o inmigrantes pineros ${ }^{5}$ ) se mueven en un continuum de variación que oscila entre un modelo de Seseo-Ceceo (I) y un modelo de DistinciónReducción (II). En el primero (I) se presentan tres pautas basadas en tres diferentes tablas de probabilidades condicionadas: $\mathrm{P}$ [seseo] $(\mathrm{s}>\mathrm{s}, \mathrm{z}>\mathrm{s}) ; \mathrm{P}$ [ceceo] $(\mathrm{z}>\theta ; \mathrm{s}>\theta)$ y $\mathrm{P}$ [mantenimiento] $(\mathrm{z}>\theta, \mathrm{s}>\mathrm{s})[\ldots]$; en el modelo de Distinción-Reducción (II), se distinguen, solo dos pautas bien diferenciadas (Villena y Requena, 1996: 12-14):

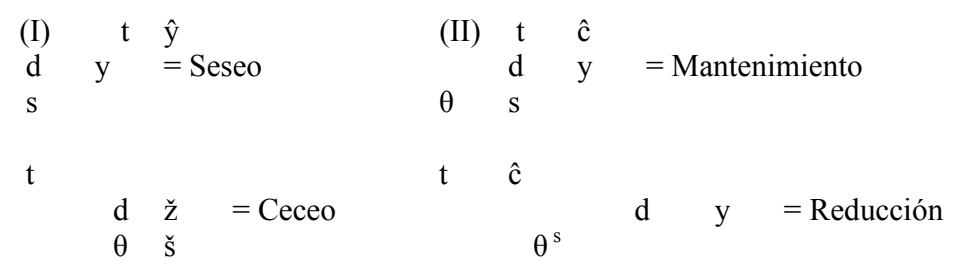

El prestigio social es uno de los factores condicionantes de ese gradata de variación. La conducta lingüística de los hablantes se organiza bien en función de un prestigio patente (denominado overt prestige o abierto), bien en función de un prestigio latente (denominado covert prestige o encubierto). El primero se relaciona con lo institucional o formal; el segundo, con la vida diaria y las relaciones informales de los individuos. Así pues, se constata la existencia de dos polos opuestos en este continuo de variación lingüística: (a) por un lado, las variedades vernaculares $(\mathrm{V})$, que utilizan los hablantes en relación a su prestigio encubierto; y, (b) por otro, la variedad estándar o ejemplar (E) íntimamente relacionada con el prestigio abierto.

\footnotetext{
${ }^{5}$ Denominamos hablantes rurales a aquellos sujetos que pertenecen a la muestra de población residente en Pinos Puente e inmigrantes pineros a los que proceden de dicha localidad y se han instalado definitivamente en Granada.
} 


\section{4. análisis}

\subsection{Datos generales}

Para el análisis de los datos se procede a su codificación mediante el programa estadístico SPSS. Se realiza un estudio de probabilidades de ambas muestras con el fin de establecer las diferencias fundamentales entre ellas.

Esta investigación cuenta con una amplia tradición en los estudios meridionales (tabla 1):

\begin{tabular}{|c|c|c|c|}
\hline & SESEO & CECEO & DISTINCIÓN \\
\hline Sevilla & 0.87 & 0.06 & 0.07 \\
\hline Jerez & 0.44 & 0.47 & 0.09 \\
\hline Huelva & 0.06 & 0.23 & 0.71 \\
\hline Córdoba & 0.51 & 0.00 & 0.49 \\
\hline Málaga & 0.31 & 0.25 & 0.70 \\
\hline Granada & 0.40 & 0.05 & 0.55 \\
\hline Pinos Puente & 0.03 & 0.70 & 0.17 \\
\hline Inmigrantes granadinos & 0.12 & 0.36 & 0.66 \\
\hline
\end{tabular}

Tabla 1. Probabilidades de seseo, ceceo y distinción de s/ $\theta$ en el español andaluz. Fuente: Sevilla (Carbonero, 1982, 1985); Jerez (Carbonero et alii, 1992); Huelva (de las Heras et alii, 1996); Córdoba (Uruburu, 1990); Málaga (Ávila, 1994); Granada (Moya y García Wiedemann, 1995)

La situación sociolingüística que manifiesta la variable $/ \Theta^{\mathrm{s}} /$ en su realización mate (сесео) o estridente (seseo) no ha resultado ajena a los investigadores, de ahí que haya sido tratada desde antiguo (Navarro Tomás, 1933; Zamora Vicente, 1960; Alvar, 1973). Los fenómenos de confusión, desfonologización (Carbonero, 1982), neutralización (ALEA), igualación fonológica (Llorente, 1962) o reducción (Sawoff, 1980) han sido profundamente analizados. Sin embargo, son pocos los estudios cuantitativos sincrónicos que han atendido a este problema. Tras el trabajo de Navarro Tomás (1933) sobre la frontera del andaluz, Alvar (1973, 1974) y Llorente (1962) se dedicaron a estudiar este asunto delimitando en el ALEA las áreas fonéticas de variación $/ \theta^{\mathrm{s}} \%$

En Sevilla, Carbonero $(1982,1985)$ se ocupó del estudio de reducción de $/ \mathrm{s} /=/ \theta /$ junto a otros fenómenos. Sus resultados revelan una alta aceptación del seseo $(0.87)$ frente a la distinción o el ceceo, considerado éste último una norma de pronunciación popular. Por su parte, en Jerez, Carbonero y colaboradores (1992) observaron que el ceceo (0.47) alternaba con el seseo (0.44) y la distinción estaba siendo patrocinada por los hablantes más jóvenes de la población.

Por su parte, de las Heras et alii (1996) estudia la variación de $/ \theta^{\mathrm{s}} /$ en la provincia de Huelva. Los resultados muestran un índice no muy alto de ceceo (.23); e igual ocurre en Málaga (.25) (Ávila, 1994) y Granada (Moya y García Wiedemann, 1995), debido al escaso 
prestigio social de que goza la realización mate (ceceo). Esta pronunciación es propia de hablantes con escaso grado de instrucción formal frente a la distinción que suele ser característica de jóvenes con estudios universitarios. Por tanto, los hablantes de las tres provincias mencionadas se inclinan a favor de la pronunciación canónica (distinción de /s/: /9/): .71 (Huelva); .70 (Málaga) y .55 (Granada).

En Córdoba, Uruburu (1990) estudia el seseo para establecer los condicionamientos sociales del fenómeno. Su investigación se centra en hablantes jóvenes con estudios medios y preuniversitarios. Sus datos revelan un 0.51 de seseo frente a un 0.49 de distinción. Por tanto, el seseo parece que no es un rasgo prestigioso en el grupo investigado puesto que alternan las realizaciones reductoras (seseantes) con las distinguidoras.

Ávila (1994), por su parte, estudió una red social de hablantes del barrio de Capuchinos de Málaga ${ }^{6}$ y contabilizó los trueques de sibilantes (seseo y ceceo) frente a los casos de conservación o mantenimiento de $\mathrm{s}$ y z. Los resultados indicaron un alto porcentaje de distinción (0.70) frente un 0.25 de ceceo y 0.31 de seseo. Este último fenómeno era propio de las mujeres frente al ceceo que era la opción elegida entre los hombres.

En Granada, el ceceo carece de prestigio y no sólo cuenta con escaso apoyo social sino que además es un rasgo estigmatizado como rural (Moya y García Wiedemann, 1995). Esta situación hace que los inmigrados procedentes de áreas de ceceo, como es el caso de nuestros informantes, modifiquen su conducta lingüística y adopten otras formas más prestigiosas. En la capital granadina, las variedades de prestigio son la distinción (0.55) y el seseo (0.40).

Ahora bien, si observamos los datos obtenidos en las calas objeto de estudio (Pinos Puente y Granada) hemos de apuntar algunas diferencias:

a) En el pueblo (Pinos Puente) la norma de prestigio (encubierto) es el ceceo (0.70). Recordemos que esta localidad está ubicada en un área de ceceo (ALEA, mapa 1705, «Áreas de mantenimiento o de neutralización de la oposición $/ \mathrm{s} /: / \theta / »)$, por ello, los hablantes residentes en Pinos Puente prefieren este fenómeno. La norma distinguidora presenta una probabilidad baja (0.17) ya que es únicamente adoptada por individuos jóvenes o mayores de cincuenta y cuatro años con estudios superiores (universitarios, sobre todo). El seseo, por su parte, apenas tiene importancia entre los hablantes y cuando aparece, es por confusión (0.03).

b) En Granada, los inmigrantes procedentes de Pinos Puente cambian completamente su actuación lingüística en beneficio de la norma distinguidora (0.66). La distinción es un fenómeno que está cobrando relieve en la capital granadina patrocinado por hablantes jóvenes con un alto nivel educativo. En nuestro caso, este patrón estándar está siendo promovido por jóvenes universitarios y hablantes de la tercera generación con estudios superiores. No obstante, el ceceo aún sigue teniendo un peso importante. La norma vernácula $(0.36)$ está presente en todas las generaciones aunque es el patrón antiestándar mayoritario de los hablantes de la tercera generación con escaso

\footnotetext{
${ }^{6}$ Del mismo modo hemos de tomar en consideración los datos de Villena (1996). En su estudio, llega a la conclusión de que la distinción de /s/: / $/$ / es el fenómeno lingüístico que goza de mayor prestigio (.62). Asimismo advierte que el ceceo es secundario y propio de hablantes con escaso grado de instrucción y el seseo resulta poco significativo (.11).
} 
grado de instrucción. Por lo que respecta al seseo, conviene recordar que los hablantes inmigrados lo articulan en un intento fallido por adquirir la distinción /s/:/ $\theta$ / como norma de prestigio de la capital. En ese intento de adquisición de la dorsal /s/, producen las realizaciones seseantes.

\subsection{Edad}

Un estudio generacional del fenómeno de variación $/ \theta^{\mathrm{s}} /$ nos proporciona datos significativos sobre el funcionamiento del ceceo, seseo y distinción en las calas de población investigadas. En la tabla 2 se exponen los resultados obtenidos para PS, PC y PD en el volumen total de la muestra recogida (pueblo y Granada):

\begin{tabular}{|lcccccc|}
\hline Generaciones & \multicolumn{2}{c}{ PS } & \multicolumn{2}{c}{ PC } & \multicolumn{2}{c|}{ PD } \\
& P.Pte $^{7}$ & Granada & P.Pte & Granada & P.Pte & Granada \\
\hline $1^{\text {a } \text { Generación }}$ & 0.00 & 0.12 & 0.67 & 0.23 & 0.22 & 0.72 \\
$2^{\text {a Generación }}$ & 0.01 & 0.11 & 0.76 & 0.37 & 0.13 & 0.66 \\
$3^{\text {a } \text { Generación }}$ & 0.10 & 0.12 & 0.68 & 0.47 & 0.16 & 0.61 \\
& & & & & & \\
\hline
\end{tabular}

Tabla 2. Probabilidades de seseo (PS), ceceo (PC) y distinción (PD) según generaciones en Pinos Puente y Granada

Se pone de manifiesto una trayectoria distinta para los fenómenos estudiados. En Pinos Puente, la distinción de /s/:/ $\theta$ / está presente en los tres grupos generacionales; sin embargo, es adoptada en mayor medida por los jóvenes (0.22) y hablantes de la tercera generación (mayores de 54 años). El ceceo es la norma vernácula de los sujetos del pueblo, por esto, es la realización mayoritaria en la cala investigada. Sorprende, no obstante, que alcance una probabilidad alta en los hablantes de edades intermedias y no en los individuos de la tercera generación cuyo índice de ceceo es prácticamente idéntico al de los jóvenes. Hemos de pensar que la segunda generación de hablantes abarca un sector de población bastante amplio (25- 54 años) que comprende individuos de casi dos generaciones distintas, por esto quizá la probabilidad de aparición de la norma reductora (ceceo) supere a la de las otras generaciones. En último lugar, el seseo, apenas tiene peso en la muestra recogida ya que, como indicábamos anteriormente, es el resultado de los continuos intentos fallidos de adquisición de la distinción.

Los datos arrojan unos índices completamente diferentes en el caso de los inmigrantes pineros. El ceceo funciona de forma inversa a la distinción. Así pues, mientras la norma reductora ceceante se correlaciona positivamente con la edad, es decir, hay un incremento del ceceo conforme avanzan las generaciones; la distinción, como norma estándar de prestigio, es patrocinada por los jóvenes principalmente, aunque aparece en todos los grupos generacionales. El seseo, por último, aunque ha crecido notoriamente con respecto a los

\footnotetext{
${ }^{7}$ Utilizaremos esta abreviatura para referirnos a los hablantes residentes en Pinos Puente y la denominación Granada para hacer mención a los inmigrantes procedentes del pueblo que viven en la capital.
} 
hablantes del pueblo de cualquier generación, sin embargo no constituye la norma prioritaria. De nuevo, hemos de entender la aparición de realizaciones seseantes como articulaciones confundidoras de los inmigrantes en su intento de lograr la distinción granadina.

Las diferencias entre los informantes del pueblo y Granada son evidentes: el ceceo ha perdido protagonismo (aunque éste aún sigue teniendo importancia en todas las generaciones) a favor de la distinción que está siendo promovida por jóvenes, sobre todo. El seseo se interpreta de igual forma en las dos muestras de habla recogidas: falta de distinción sistemática entre $/ \mathrm{s} / \mathrm{y} / \theta /$.

\subsection{Educación}

El nivel educativo del hablante incide directamente sobre los fenómenos investigados. En las líneas que siguen estableceremos una comparación entre los datos obtenidos en el pueblo y en Granada con el objeto de determinar la influencia de esta variable social. En la tabla 3 se exponen los resultados generales según el grado de instrucción de los hablantes:

\begin{tabular}{|llccccc|}
\hline Educación & \multicolumn{2}{c}{ PS } & \multicolumn{2}{c}{ PC } & \multicolumn{2}{c|}{ PD } \\
& P.Pte & Granada & P.Pte & Granada & P.Pte & Granada \\
\hline Sin estudios & 0.00 & 0.18 & 0.92 & 0.58 & 0.01 & 0.59 \\
Estudios Medios & 0.02 & 0.09 & 0.75 & 0.31 & 0.09 & 0.64 \\
Estudios Superiores & 0.10 & 0.05 & 0.31 & 0.08 & 0.53 & 0.84 \\
\hline
\end{tabular}

Tabla 3. Índices obtenidos según educación de los hablantes residentes en Pinos Puente y Granada

De la actuación lingüística de los hablantes residentes en el pueblo, podemos extraer algunas conclusiones:

1) El ceceo muestra una clara estratificación educacional ya que conforme disminuye el nivel educativo del hablante se incrementa el número de realizaciones ceceantes. Son, por tanto, los sujetos sin ningún grado de instrucción los que presentan los índices más altos de ceceo.

2) La norma distinguidora se comporta de forma inversa al ceceo puesto que crece la probabilidad de distinción conforme aumenta la educación del hablante.

3) El seseo, a pesar del poco valor que representa para el volumen total de la muestra, se manifiesta mínimamente en los informantes con estudios superiores porque son ellos principalmente los que tratan de acomodarse a la distinción de /s/: / $/ \theta$. En ese proceso de adaptación producen articulaciones seseantes en mayor medida que el resto de sujetos. Por tanto, la educación constituye un factor decisivo de la actuación lingüística de los hablantes: a mayor nivel educativo, más posibilidades de aparición de la norma estándar (distinción) y, al contrario, a menor grado de instrucción, mayores posibilidades de conservación del ceceo. 
Una situación similar ofrecen los hablantes procedentes de Pinos Puente que residen en Granada; si bien conviene precisar algunos matices:

1) El ceceo ha reducido notablemente su índice de aparición, $(0.58=$ inmigrantes sin estudios / 0.92 = hablantes de Pinos Puente sin estudios) aunque aún sigue teniendo importancia entre los inmigrantes puesto que es su norma vernácula. De este modo, se da una estratificación esperada del fenómeno puesto que desciende el ceceo a medida que se incrementa el nivel educativo del hablante.

2) La distinción, por el contrario, se ha extendido considerablemente a todos los sujetos, con independencia de su grado de instrucción. Recordemos que en los hablantes residentes en el pueblo apenas tenía importancia, salvo en los informantes con estudios superiores. A diferencia del ceceo, la distinción crece conforme aumenta la educación del sujeto.

3) El seseo ha cambiado su funcionamiento con respecto a lo que ocurría en el pueblo. Ahora son los hablantes sin estudios los que presentan una probabilidad más alta de seseo (0.18). Si interrelacionamos la educación con las generaciones arriba estudiadas (tabla 2), podemos comprobar que el seseo obtenía unos índices similares en los tres grupos generacionales $(0.12 ; 0.11 ; 0.12$ respectivamente). Los datos que nos proporciona la educación nos permite afirmar que son los jóvenes sin estudios los que patrocinan esta norma reductora (seseo). En la muestra de habla constituida por inmigrantes granadinos instalados, este sector de población (jóvenes sin estudios) es muy heterogéneo ya que está compuesto por hijos de padres procedentes de Pinos Puente que han nacido en la capital granadina, influenciados por varios factores: la variedad lingüística hablada por sus padres (ceceo, seseo o distinción), el contacto con amigos o familiares del pueblo, la educación, el barrio de residencia, etc. Todas estas variables han podido influir en la conformación de su variedad lingüística.

En suma, podemos decir que:

- El ceceo es propio de hablantes sin estudios, con independencia del lugar de residencia (pueblo o Granada). Es importante el descenso del índice de ceceo entre los inmigrantes granadinos, habida cuenta del prestigio de la distinción $/ \mathrm{s} /: / \theta /$.

- La norma distinguidora crece conforme aumenta el nivel educativo de los inmigrantes instalados, aunque ya se extiende a todos los grupos investigados. En el pueblo, dado el prestigio encubierto de que goza el ceceo, la distinción es la pauta de comportamiento elegida únicamente por los sujetos con estudios superiores.

- El seseo pasa de ser propio de hablantes del pueblo con un nivel educativo elevado a ser la variedad lingüística preferida por los inmigrantes granadinos sin ningún grado de instrucción formal. Las especiales características de este grupo (jóvenes sin estudios) hacen posible el cambio. 


\subsection{Sexo}

Desde el punto de vista de las probabilidades obtenidas de ceceo (PC) y distinción (PD), sobre todo, existen diferencias importantes entre los hablantes del pueblo y Granada. Veamos a continuación qué ocurre en cada caso (tabla 4):

\begin{tabular}{|lcccccc|}
\hline Sexo & \multicolumn{2}{c}{ PS } & \multicolumn{2}{c}{ PC } & \multicolumn{2}{c|}{ PD } \\
& P.Pte & Granada & P.Pte & Granada & P.Pte & Granada \\
\hline Hombre & 0.04 & 0.12 & 0.74 & 0.33 & 0.15 & 0.62 \\
Mujer & 0.03 & 0.11 & 0.66 & 0.38 & 0.19 & 0.71 \\
\hline
\end{tabular}

Tabla 4. Diferencias por sexos entre los hablantes residentes en Pinos Puente y los inmigrantes granadinos instalados

En relación a los hablantes residentes en Pinos Puente, se observa que los hombres muestran una alta probabilidad de ceceo $(0.74$, frente a 0.66 en las mujeres). Sin embargo, las féminas aunque son ceceantes, están iniciando un cambio hacia la distinción de /s/:/ $\theta /$ ( 0.19 / 0.15 en los hombres). Normalmente, se trata de mujeres con un alto nivel educativo cuya profesión les ha permitido establecer contactos fuera del pueblo. Esto ha generado un intento de adquisición de la norma estándar (distinción). En cuanto, a la probabilidad de seseo, observamos que las diferencias genéricas son prácticamente inexistentes, dada la escasa importancia de que goza esta norma reductora en la comunidad de habla estudiada.

La variación según sexos cambia cuando estos hablantes procedentes de Pinos Puente se instalan en Granada: en primer lugar, se produce un descenso considerable de la norma reductora (ceceo) en ambos grupos ( 0.33 hombres, frente a 0.38 en las mujeres), dado el prestigio de la distinción en la capital granadina. Tanto hombres como mujeres tratan de abandonar su ceceo aunque no lo consiguen plenamente ya que constituye su norma vernácula. Ellas optan por esta norma reductora de escaso prestigio social, con una diferencia de apenas 5 puntos con respecto a los hombres; en segundo lugar, advertimos que la probabilidad de distinción es más alta en las mujeres (0.71) que en los hombres (0.62). Las hablantes del sexo femenino suelen ser más innovadoras y receptivas a los cambios de ahí que se decanten, en mayor medida, por la norma que goza de prestigio social (distinción /s/: / $/$ /); por último, el seseo, aunque ha extendido su influencia entre los inmigrantes instalados, no se aprecian grandes diferencias entre hombres $(0.12)$ y mujeres (0.11). Los hablantes de ambos sexos tratan de acomodarse a la norma culta granadina (distinción) y, en ese intento, se incrementa el número de realizaciones seseantes por la ausencia de la dorsal /s/ en su sistema fonológico.

En el Gráfico 1 corroboramos el diferente comportamiento lingüístico que adoptan los hablantes de Pinos Puente con respecto a las normas de ceceo y distinción. Tanto hombres como mujeres se decantan por el ceceo, en su mayoría; sin embargo, existen algunos individuos que prefieren la distinción, de ahí que haya algunos puntos en el gráfico muy cercanos a esta norma estándar. 


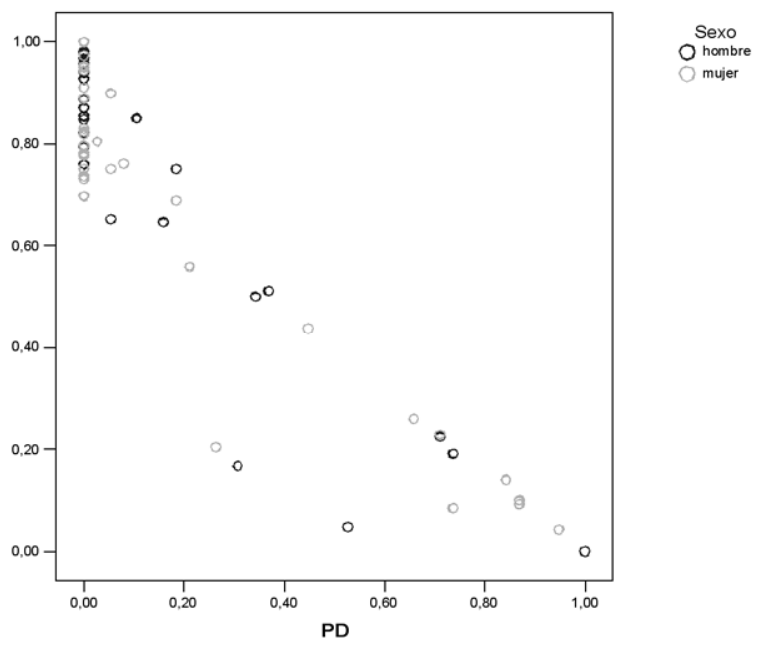

Gráfico 1

Dispersión de los hablantes de Pinos Puente en el continuo probabilístico de PC y PD según sexo

Por el contrario, el Gráfico 2 revela el cambio de actitud que han manifestado los sujetos investigados tras instalarse definitivamente en la capital granadina. El gráfico muestra que la distinción ha crecido notoriamente tanto en hombres como en mujeres; por el contrario, el ceceo ha reducido su influjo aunque no está ausente de las realizaciones de los inmigrantes. Por esto, aún aparecen en la figura que mostramos varios puntos dispersos en el eje Y. El resto de hablantes bien sesean, bien producen articulaciones intermedias de

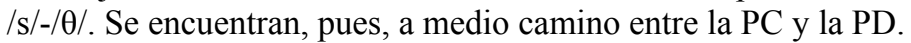




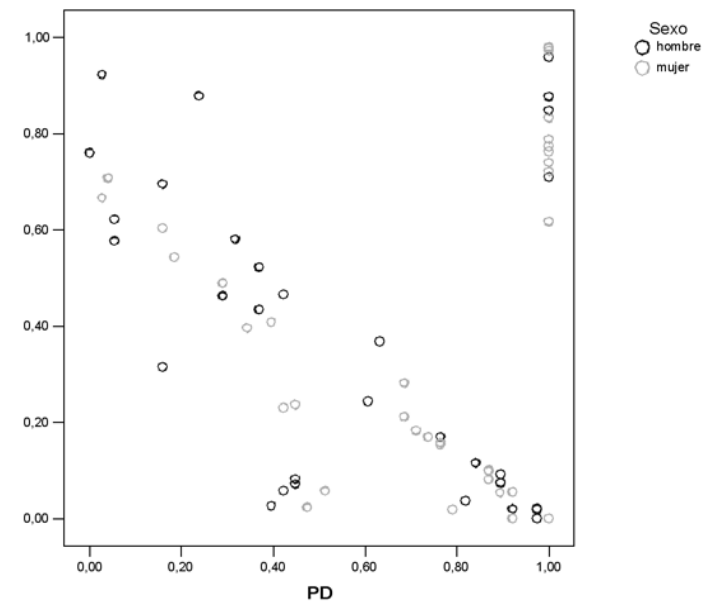

Gráfico 2

Dispersión de los inmigrantes pineros en el continuo probabilístico de PC y PD según sexo

En suma, advertimos que tanto los hombres como las mujeres del pueblo adoptan en su mayoría el ceceo puesto que es su norma vernácula. Notamos, no obstante, una tendencia en ambos sexos hacia la distinción, siendo algo mayor en las mujeres (0.19) que en los hombres (0.15). El seseo apenas tiene repercusión. En Granada, los inmigrantes cambian su patrón de comportamiento. Ahora el ceceo pierde importancia en beneficio de la norma distinguidora. La distinción está patrocinada por mujeres principalmente $(0.71$, frente a 0.62 de los hombres) y el seseo, alcanza algo de protagonismo cuando los sujetos inmigrados de ambos sexos no logran adquirir satisfactoriamente la ansiada distinción de $/ \mathrm{s} /: / \theta /$.

\section{5. análisis de correlaciones}

En última instancia, interesa conocer las correlaciones que existen entre las variables lingüísticas con el objeto de comprobar el distinto funcionamiento de los fenómenos investigados. Se procedió pues, a la realización de un análisis de correlaciones entre la PS, PC y PD para las dos calas de población investigadas. De este análisis se desprenden datos significativos:

1) Los hablantes residentes en Pinos Puente (tabla 5) presentan: (a) una correlación muy alta entre la PD y la PC: $\mathrm{R}=-, 928$ en sentido negativo. Esto significa que a medida que aumenta el ceceo, disminuye la distinción; (b) una correlación negativa media entre la PS y la $\mathrm{PC}: \mathrm{R}=-, 403$. Estos datos revelan que los residentes en Pinos Puente o sesean o cecean pero no tiene lugar la mezcla. Así lo 
hemos justificado a lo largo de este estudio; (c) una correlación positiva muy baja entre la PD y PS: $R=$,135. Esto indica que ambas variables no están correlacionadas. Es muy difícil, por no decir imposible, encontrar en el pueblo hablantes distinguidores y seseantes al mismo tiempo.

\begin{tabular}{|c|c|c|c|c|}
\hline & & Pceceo & Pseseo & Pdistinción \\
\hline \multirow[t]{3}{*}{ Pceceo } & Correlación de Pearson & 1 &,$- 403(* *)$ &,$- 928(* *)$ \\
\hline & Sig. (bilateral) & &, 000 & ,000 \\
\hline & $\mathrm{N}$ & 72 & 72 & 72 \\
\hline \multirow[t]{3}{*}{ Pseseo } & Correlación de Pearson &,$- 403(* *)$ & 1 & 135 \\
\hline & Sig. (bilateral) & ,000 & & 259 \\
\hline & $\mathrm{N}$ & 72 & 72 & 72 \\
\hline \multirow[t]{3}{*}{ Pdistinción } & Correlación de Pearson &,$- 928(* *)$ & 135 & 1 \\
\hline & Sig. (bilateral) &, 000 & 259 & \\
\hline & $\mathrm{N}$ & 72 & 72 & 72 \\
\hline
\end{tabular}

Tabla 5. Correlaciones bivariadas en los hablantes residentes en Pinos Puente ** La correlación es significativa al nivel 0,01 (bilateral)

2) Los inmigrantes pineros obtienen datos completamente diferentes (tabla 6): (a) una correlación negativa baja entre la PD y la PC: $\mathrm{R}=-, 198$. Esto indica que ambos fenómenos no están muy correlacionados, es decir, que los individuos bien optan por el ceceo, bien optan por la distinción. A pesar de ello, la correlación se da en sentido negativo, lo cual manifiesta que a menor ceceo, mayor es la distinción y viceversa; (b) una correlación también baja y negativa entre la PS y $\mathrm{PD}: \mathrm{R}=-$,297. Los hablantes sesean cuando intentan distinguir $\mathrm{s} / \mathrm{z}$; (c) la correlación entre la PS y PC es algo más baja que la anterior: $\mathrm{R}=-, 230$. Por tanto, se puede decir que no existe correlación entre ambas variables. El ceceo es un fenómeno que funciona de forma distinta al seseo, tal y como hemos comprobado a lo largo de este trabajo. 


\begin{tabular}{|c|c|c|c|c|}
\hline & & Pceceo & Pseseo & Pdistinción \\
\hline \multirow[t]{3}{*}{ Pceceo } & Correlación de Pearson & 1 &,- 230 &,- 198 \\
\hline & Sig. (bilateral) & & ,052 & ,096 \\
\hline & $\mathrm{N}$ & 72 & 72 & 72 \\
\hline \multirow[t]{3}{*}{ Pseseo } & Correlación de Pearson &,- 230 & 1 &,$- 297(*)$ \\
\hline & Sig. (bilateral) & 052 & & 011 \\
\hline & $\mathrm{N}$ & 72 & 72 & 72 \\
\hline \multirow[t]{3}{*}{ Pdistinción } & Correlación de Pearson &,- 198 &,$- 297(*)$ & 1 \\
\hline & Sig. (bilateral) & ,096 &, 011 & \\
\hline & $\mathrm{N}$ & 72 & 72 & 72 \\
\hline
\end{tabular}

Tabla 6. Correlaciones bivariadas en los inmigrantes pineros

* La correlación es significante al nivel 0,05 (bilateral)

** La correlación es significativa al nivel 0,01 (bilateral)

\section{6. conclusiones}

De esta investigación podemos extraer las siguientes conclusiones:

1) Los datos generales apuntan que en Pinos Puente hay un predominio de la norma vernácula (ceceo) en detrimento del seseo o la distinción. Si bien el seseo apenas tiene relevancia, la distinción está siendo patrocinada por hablantes con estudios superiores. Por el contrario, en Granada los inmigrantes pineros se decantan por la distinción como norma culta de prestigio. No obstante, el ceceo aún sigue teniendo un peso importante. Por su parte, el seseo se incrementa. Hay una actitud proclive de los hablantes hacia la adquisición de la distinción granadina $\mathrm{y}$, por tanto, los intentos fallidos por conseguirla son mayores. El análisis de determinados factores sociales en el seno de los dos grupos de individuos analizados demuestra, en suma, que la distinción estándar del español entre $/ \mathrm{s} / \mathrm{y} / \theta /$ es un cambio prestigioso entre los inmigrantes instalados en Granada que está sustituyendo a la reducción fonológica $(/ \mathrm{s} /=/ \theta /)$ que, aunque es notoria aún entre los inmigrados, sin embargo, cobra relieve en los individuos de procedencia rural (residentes en Pinos Puente).

2) La edad permite profundizar en las diferencias entre la metrópoli (Granada) y su área metropolitana (Pinos Puente). El ceceo sigue ejerciendo su influencia en los hablantes pineros de cualquier generación. Sin embargo, en Granada muestra una estratificación normal ya que se incrementa conforme aumenta la edad de los inmigrantes. La distinción está patrocinada por jóvenes, tanto en el pueblo como en Granada; y, el seseo se manifiesta en los inmigrantes de cualquier generación, si bien en el pueblo predominaba entre los mayores de cincuenta y cuatro años.

3) La educación constituye un factor importante en esta investigación. Tanto en Pinos Puente como en Granada el ceceo aumenta conforme disminuye el grado de instrucción de los hablantes, aunque en el pueblo la probabilidad es mucho más 
alta. La distinción funciona de forma inversa al ceceo y se correlaciona positivamente con la instrucción: crece a medida que los estudios del informante son más elevados. El seseo, en cambio, pasa de ser propio de hablantes pineros con estudios superiores a inmigrantes granadinos sin estudios ${ }^{8}$.

4) Las diferencias por sexos son destacadas. En el pueblo, existen dos normas de pronunciación fundamentales: el ceceo y la distinción. Los hombres optan por el ceceo en su mayoría, al igual que las mujeres. La distinción, aunque minoritaria, se da en ambos sexos pero está promovida por mujeres principalmente. En Granada entra en juego una tercera norma: el seseo. Ahora, los inmigrantes granadinos de procedencia rural prefieren la distinción (más elevada en mujeres que en hombres); seguida del ceceo, que aún sigue teniendo peso; y el seseo, que incrementa su índice en ambos sexos?.

5) En suma, podemos hablar de la existencia de dos modelos de variación lingüística (3.2.). Tras la investigación hemos comprobado que los hablantes de las dos calas de población investigadas se mueven en un continuum de variación que oscila entre los rasgos vernaculares (ceceo) y la norma estándar granadina (distinción /s/: /Ө/): (a) en Pinos Puente existe una variedad vernácula concentrada (ceceo) que se refuerza por las redes sociales densas que mantienen muchos de sus hablantes. Sin embargo, estas redes sociales se van aflojando cuando los sujetos emigran a la capital granadina, puesto que su variedad vernácula entra en contacto con otras variedades urbanas. Esto propicia por un lado, una convergencia horizontal por la aproximación entre las variedades vernaculares (coinización): se conserva el ceceo o aparece el seseo por confusión sistemática; y, por otro, una convergencia vertical en el sentido de la variedad estándar (nivelación). La solución es la adopción de la distinción de /s/: / $/$ /; (b) cuando estos sujetos se han instalado en la capital granadina se decantan bien por la reducción $/ \theta^{\mathrm{s}} /$ (modelo I): el ceceo normalmente se manifiesta entre los jóvenes o mayores sin estudios aunque no está ausente en el resto de los grupos; bien por la distinción (modelo II): hablantes jóvenes o mayores con un alto nivel educativo; La falta de instrucción formal impide a ambas generaciones adoptar la norma culta granadina (la distinción); el seseo, por su parte, aparece por confusión de los hablantes en un intento de adquirir la distinción $\operatorname{de} / \mathrm{s} /: / \theta /$.

\footnotetext{
${ }^{8}$ En Granada los jóvenes sin estudios eligen la norma seseante influenciados por varios factores: barrio de residencia en la ciudad, educación, variedad lingüística de sus padres o amigos, etc. En el pueblo, en cambio, son los mayores con estudios superiores, en su intento de lograr la distinción, los que producen articulaciones seseantes. Esta inquietud no se da en los jóvenes que o bien, son ceceantes o bien, distinguidores.

${ }^{9}$ Existe una preocupación mayor entre los inmigrantes granadinos por adquirir la norma culta que goza de prestigio

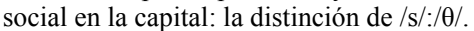




\section{Referencias bibliográficas}

Alvar, M.; A. Llorente y G. Salvador (1960-1973): Atlas Lingüístico y Etnográfico de Andalucía, IVI, Universidad de Granada-CSIC, ed. Facsímil, I (I-II), II (III-IV), III (V-VI). Madrid: ArcoLibros.

Alvar, M. (1973): Notas de asedio al habla de Málaga, Tirada Aparte de Málaga, 16 y 17. Málaga: Ayuntamiento de Málaga.; reed. en Puerta Nueva, Revista de Educación, Consejería de Educación de la Junta de Andalucía, 17, marzo, 1973, págs. 6-13.

Alvar, M. (1974): "Sevilla, macrocosmos lingüístico. Fonética y fonología según el Atlas Lingüístico y Etnográfico de Andalucía”. En Homenaje a Ángel Rosenblat en sus 70 años. Caracas: Instituto Pedagógico, págs. 13-42.

Ávila Muñoz, A. (1994): La variación reticular e individual en el vernáculo urbano malagueño. Datos del barrio de Capuchinos. Memoria de Licenciatura Inédita, Departamento de Filología Española I, Universidad de Málaga.

Carbonero, P. (1982): "Norma estándar y actitud sociolingüística". En Carbonero, P. (1985): Sociolingüística andaluza. Metodología y Estudios. Universidad de Sevilla, ${ }^{2} 1985$ [1982], págs. 141-150.

Carbonero, P. et alii (1992): El habla de Jerez. Estudio sociolingüístico, BUC, Cuadernos de Divulgación. Jerez: Ayuntamiento de Jerez.

Heras, J. et al. (1996): "Perfil sociolingüístico del habla culta de la zona periurbana de Huelva", Aestuaria, IV-4, págs.109-124.

Labov, W. (1984): "Field methods of the Project of linguistic change and variation". En J. Baugh y J. Sherzer (eds.) (1984): Language in use. Readings in sociolinguistics. Englewood Cliffs, N.J.: Prentice-Hall, págs. 28-53.

Llorente Maldonado, A. (1962): "Fonética y fonología andaluzas", Revista de Filología Española, 45, págs.227-240.

López Morales, H. (1994): Métodos de investigación lingüística. Salamanca: Ediciones El Colegio de España.

Milroy, L. (1987): Observing and analysing natural language. Oxford: Blackwell.

Moya Corral, J. A. y García Wiedemann, E. (1995): El habla de Granada y sus barrios. Granada: Universidad.

Navarro Tomás, T. et alii (1933): "La frontera del andaluz". En Navarro Tomás (1975): Capítulos de Geografía Lingüística de la Península Ibérica. Bogotá: Publicaciones del Instituto Caro y Cuervo, págs.20-80.

Sawoff, A. (1980): "A sociolinguistc appraisal of the sibilant pronunciation in the city of Seville", Festgabe für Norman Denison, Grazer Linguistische Studien 11-12, págs.238-262.

Uruburu, A. (1990): "Seseo en el habla juvenil de la ciudad de Córdoba (España)", Estudios sobre la lengua española en Córdoba. Córdoba: Diputación Provincial, págs.125-134.

Villena Ponsoda, J. A. (1996): "Convergence and divergente in a standard-dialect continuum: Networks and individuals in Málaga", Sociolingüística, 10. Tübingen: Max Niemeyer Verlag, págs.112-137.

Villena Ponsoda, J. A. y Requena Santos, F. (1996): "Género, educación y uso lingüístico: la variación social y reticular de s y z en la ciudad de Málaga", Lingüística, 8, págs.5-48.

Zamora Vicente, A. (1960): Dialectología española. Madrid: Gredos. 